\title{
Uma revisão sobre padrões de atividade, reprodução e alimentação de cágados brasileiros (Testudines, Chelidae)
}

\begin{abstract}
Franco L. Souza
Universidade Federal de Mato Grosso do Sul, Centro de Ciências Biológicas e da Saúde, Departamento de Biologia, 79070-900, Campo Grande, MS, Brasil. E-mail: flsouza@nin.ufms.br.
\end{abstract}

\begin{abstract}
A review on activity patterns, reproduction, and feeding habits in Brazilian chelid turtles (Testudines, Chelidae). The knowledge on natural history of some Brazilian chelonian species is still scarce what makes it difficult to ascertain ecological and evolutionary histories. Data like this, however, can be used as fundamental tools for species management and conservation. A survey was made on the activity patterns, reproduction, and feeding behavior of Brazilian Chelidae species (freshwater sidenecked turtles) available in the literature. A clear lack of information regarding natural history is evident for most species. The species diversity and the biological importance of this taxon regard it as an interesting target to be studied. Since several Chelidae behaviors (including those reported in this study) are considered stereotyped, I suggest that laboratory studies should be encouraged as a way of complementing the field research.
\end{abstract}

Keywords: Testudines, Chelidae, Acanthochelys, Batrachemys, Bufocephala, Chelus, Hydromedusa, Mesoclemmys, Platemys, Phrynops, Ranacephala, Rhinemys, activity patterns, feeding, reproduction.

Palavras-chave: Testudines, Chelidae, Acanthochelys, Batrachemys, Bufocephala, Chelus, Hydromedusa, Mesoclemmys, Phrynops, Platemys, Ranacephala, Rhinemys, alimentação, padrões de atividade, reprodução.

\section{Introdução}

Aproximadamente $20 \%$ das 278 espécies de quelônios do mundo ocorrem na América do Sul, representando oito famílias (Dermochelyidae, Cheloniidae, Chelydridae, Emydidae,

Recebido em 2 de abril de 2004.

Aceito em 26 de junho de 2004.

Distribuído em 30 de setembro de 2004.
Kinosternidae, Testudinidae, Podocnemididae e Chelidae). Dessas, a família Chelidae, cujos representantes típicos são os animais conhecidos popularmente como cágados, é a mais rica, contando com 23 espécies, das quais 19 ocorrem no Brasil (ver abaixo). O conhecimento da história natural das espécies brasileiras de cágados é bastante incipiente, apesar de estudos conduzidos desde as últimas décadas terem contribuído de maneira significativa para a elucidação de vários aspectos da biologia do 
grupo. Entretanto, a escassez de conhecimento dificulta abordagens mais amplas sobre vários aspectos ecológicos e evolutivos das espécies, que são primordiais em eventuais planos de conservação e manejo.

Este trabalho teve como objetivo elaborar uma compilação de informações sobre os padrões de atividade, aspectos reprodutivos e comportamento alimentar das espécies brasileiras de Chelidae. A sistemática do grupo seguiu Iverson (1992), com exceção do gênero Phrynops, para o qual foram adotadas abordagens recentes referentes à inclusão de espécies (Cabrera 1998, McCord et al. 2001). Dessa maneira, 19 espécies de Chelidae puderam ser consideradas para o Brasil: Acanthochelys macrocephala (Rhodin, Mittermeier e McMorris, 1984), A. radiolata (Mikan, 1820), A. spixii (Spix, 1824), Batrachemys helliostema McCord, Joseph-Ouni e Lamar, 2001, B. nasuta (Schweigger, 1812), B. raniceps (Gray, 1855), B. tuberculata (Luederwaldt, 1926), Bufocephala vanderhaegei (Bour, 1973), Chelus fimbriatus (Schneider, 1783), Hydromedusa maximiliani (Mikan, 1820), H. tectifera Cope, 1869, Mesoclemmys gibba (Schweigger, 1812), Phrynops geoffroanus (Schweigger, 1812), $P$. hilarii (Duméril e Bibron, 1835), P. tuberosus (Peters, 1870), P. williamsi Rhodin e Mittermeier, 1983, Platemys platycephala (Schneider, 1792), Ranacephala hogei (Mertens, 1967) e Rhinemys rufipes (Spix, 1824) (Figura 1). Sub-espécies não foram consideradas. Na medida do possível, procuraram-se exemplos cujos resultados provinham de estudos com animais encontrados em condições naturais. Eventualmente, observações de cativeiro foram consideradas. Todavia, as informações relativas a animais de cativeiro restringiram-se apenas ao comportamento de acasalamento, devido ao padrão estereotipado (Molina 1989, 1996). Por outro lado, dados reprodutivos (número de ovos) de animais mantidos em cativeiro foram desconsiderados. Como o tamanho corpóreo (comprimento da carapaça ou plastrão) de fêmeas freqüentemente restringe o número de ovos produzidos (Iverson et al. 1993), tal restrição fez-se necessária, uma vez que quelônios podem exibir crescimento e reprodução diferenciadas em ambientes com disponibilidade de recursos variável (Brown et al. 1994, Bjorndal et al. 2003, Rowe et al. 2003), algo que pode ocorrer em cativeiro. Bibliografias referentes a encontros regionais, congressos ou relatórios internos ("gray literature") foram evitadas, dado que trabalhos dessa natureza carecem de avaliação de mérito ou averiguações minuciosas e críticas.

\section{Padrões de Atividade}

Os padrões de atividade dos quelônios em geral estão intimamente associados às condições climáticas, sendo que o índice de precipitação pluviométrica e a temperatura do ar ou da água incluem-se entre os principais fatores ambientais que moldam o ritmo do comportamento das espécies. Uma vez que regimes de chuva e temperatura muitas vezes delimitam períodos sazonais, freqüentemente observa-se que as espécies exibem diferentes picos de atividade ao longo do ano. Por exemplo, para a região sul do estado de São Paulo, o cágado Hydromedusa maximiliani exibe maior atividade durante o período setembro-janeiro, justamente aquele período em que as chuvas são mais constantes, enquanto entre maio e agosto, um período caracterizado por baixas temperaturas e estiagem na região, a atividade é menor (Souza 1995, Souza e Abe 1997b). Phrynops geoffroanus é mais ativa entre setembro e novembro, estando essa atividade diretamente associada à elevada temperatura do ar, provavelmente por favorecer o comportamento de assoalhamento (Molina 1989, Souza 1999); por outro lado, a espécie é menos detectada durante períodos mais chuvosos, quando os locais disponíveis para assoalhamento ficam submersos (Souza 1999, Souza e Abe 2001). De acordo com Mittermeier et al. (1978), indivíduos de Mesoclemmys gibba mantidos em cativeiro (localidade não fornecida pelos autores) exibem 

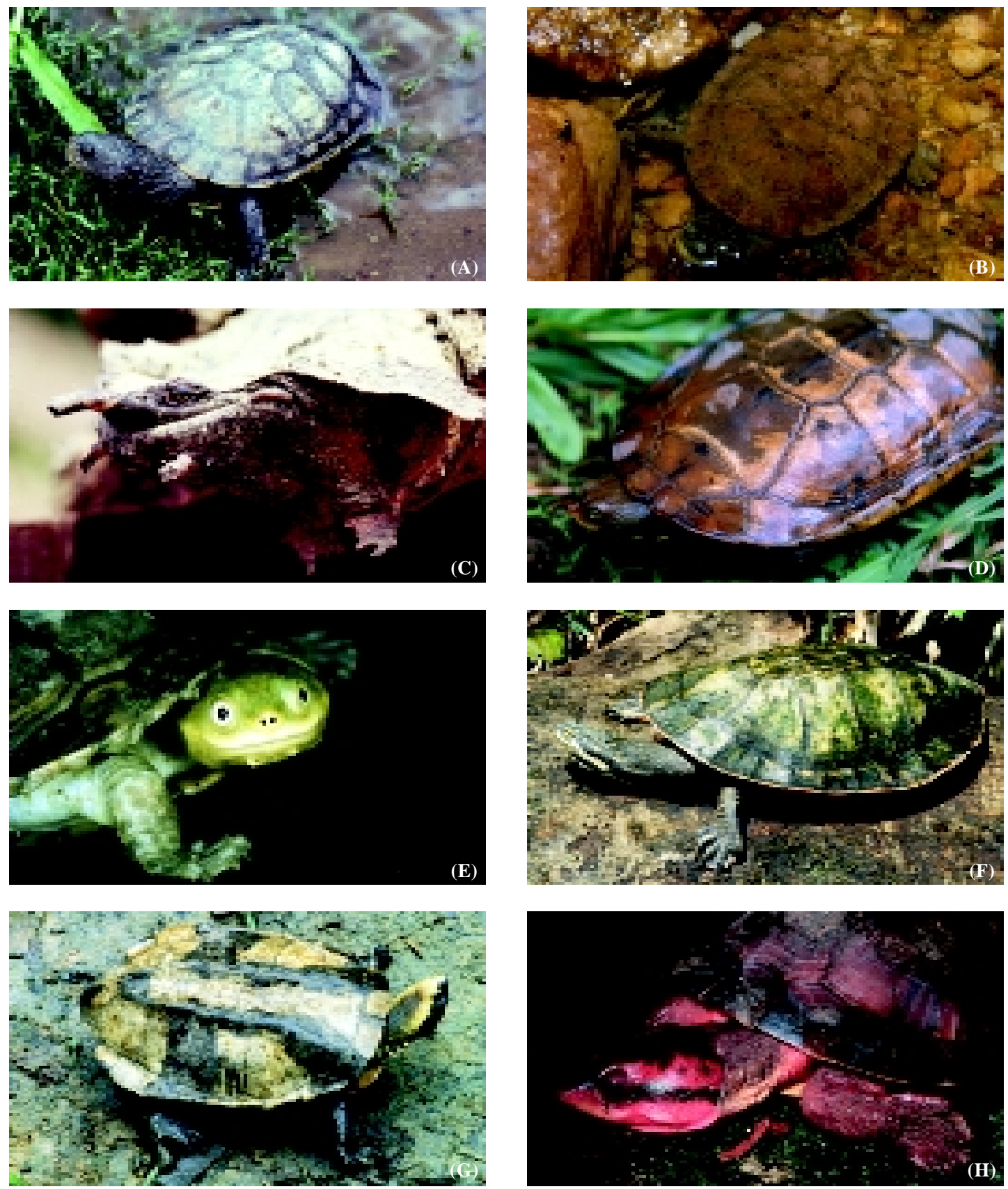

Figura 1 - Algumas espécies de Chelidae que ocorrem no Brasil. (A) Batrachemys tuberculata, Exu, PE, L. J. Vitt, (B) Bufocephala vanderhaegei, Chapada dos Veadeiros, GO, F. Toledo, (C) Chelus fimbriatus, Manaus, AM, R. C. Vogt., (D) Hydromedusa maximilliani, Serra do Caraça, MG, P. C. Eterovick, (E) Mesoclemmys gibba, Rio Ajarani, RR, L. J. Vitt, (F) Phrynops geoffroanus, Rio Formosa, RO, L. J. Vitt, (G) Platemys platycephala, P. E. Guajara Mirim, RO, L. J. Vitt, (H) Rhinemys rufipes, Manaus, AM, W. E. Magnusson 
comportamento de estivação. Como em algumas regiões colombianas de ocorrência da espécie o ambiente pode tornar-se totalmente seco durante o período de estiagem, Mittermeier et al. (1978) sugeriram que esse comportamento possa existir também na natureza. Habitando áreas de banhados na região sul do país (RS), o cágado Hydromedusa tectifera costuma enterrar-se na lama à medida que os locais tornam-se secos, comportamento também verificado durante o inverno, ressurgindo na primavera (Lema e Ferreira 1990). Acanthochelys spixii tem hábitos noturnos, tendo sido observadas migrações entre corpos d'água durante as noites de verão no Rio Grande do Sul (Lema e Ferreira 1990). Migração em busca de ambientes favoráveis também foi relatada para Batrachemys tuberculata no nordeste do Brasil (Vanzolini et al. 1980). Outra espécie de hábito noturno é $M$. gibba; porém, em cativeiro, a espécie pode, ocasionalmente, assoalhar no início da manhã ou no final da tarde (Mittermeier et al. 1978, Pritchard e Trebbau 1984). Phrynops geoffroanus e $P$. hilarii são diurnas, assoalhando durante as horas mais quentes do dia sobre troncos, pedras ou ao longo das margens dos rios (Medem 1960, Monteiro e Diefenbach 1987, Molina 1989, Souza 1999).

Hydromedusa maximiliani desloca-se pouco, em média $2 \mathrm{~m} /$ dia (Souza 1995, Souza e Abe 1997b) enquanto $P$. geoffroanus chega a mover-se $250 \mathrm{~m} / \mathrm{dia}$ (Souza 1999) e Ranacephala hogei, entre 3 e $15 \mathrm{~m} /$ dia (Moreira 2002). Na região amazônica, a área de vida estimada para Rhinemys rufipes foi de 1 a 2 quilômetros de rio, o equivalente a 0,4 a 0,8 ha (Magnusson et al. 1997). Como a dieta desse cágado é basicamente composta por frutos de palmeiras (Lima et al. 1997) (ver abaixo) e essas plantas são abundantes ao longo das margens dos rios e igarapés habitados por essa espécie, a hipótese sugerida pelos autores é a de que a distribuição espacial das palmeiras e a oferta alimentar contínua que fornecem possibilitaria essa pequena área de vida (Magnusson et al. 1997). Considerando-se essa hipótese de manutenção de uma pequena área de vida relacionada diretamente à disponibilidade de alimento, Souza (1999) sugeriu que $P$. geoffroanus deveria exibir uma área de vida menor do que aquela relatada para $R$. rufipes (Magnusson et al. 1997), uma vez que, na população de $P$. geoffroanus estudada, o alimento, em forma de esgoto doméstico, era muito abundante. A relação entre disponibilidade de alimento e área de vida ainda merece maiores estudos.

\section{Reprodução}

\section{Acasalamento}

O ritmo sazonal de atividade das espécies também está fortemente associado aos períodos reprodutivos, durante os quais os machos deslocam-se à procura das fêmeas e essas à procura de locais para nidificação. Portanto, machos e fêmeas freqüentemente exibem períodos complementares de atividade. O acasalamento parece estar restrito à água, embora Platemys platycephala ocasionalmente possa acasalar-se em terra, junto às margens dos rios (Pritchard e Trebbau 1984). O período de acasalamento é variável, dependendo da espécie. O acasalamento de Phrynops geoffroanus ocorre durante o dia, em qualquer período da manhã ou da tarde (Medem 1960, Molina 1989, 1996, 1998). Acanthochelys radiolata exibe tal comportamento à tarde (Mocelin 2001), enquanto Platemys platycephala pode acasalar-se de dia ou durante a noite (Pritchard e Trebbau 1984).

O acasalamento é um comportamento estereotipado em quelônios, em que pelo menos quatro diferentes fases de exibição podem ser identificadas. Molina (1989, 1996) forneceu detalhes minuciosos dessas fases em indivíduos de Phrynops geoffroanus mantidos em cativeiro, que são aqui resumidas: I) procura por fêmeas: os machos cheiram a cloaca de outro indivíduo com a finalidade de reconhecimento específico e determinação sexual ou para determinar a 
receptividade das fêmeas; comportamento agressivo entre machos pode ocorrer durante essa fase; II) perseguição da fêmea: os animais movem-se rapidamente, nadando ou caminhando pelo fundo dos rios, mudando de direção várias vezes (essa fase não ocorre caso a fêmea não esboce comportamento de fuga do macho que a persegue); III) pré-cópula: geralmente inicia-se quando a fêmea pára de fugir do macho ou quando o macho consegue manter-se sobre a fêmea. Durante essa fase, o macho arranha a carapaça da fêmea com as garras dos pés ou morde o pescoço da fêmea, o que funciona como um estímulo tátil. Também durante essa fase, o macho mantém o pescoço esticado e, com movimentos laterais, esfrega seu queixo contra a superfície dorsal da cabeça da fêmea. As fêmeas podem permanecer imóveis ou movimentar-se para tentar "derrubar" os machos; IV) cópula: quando a fêmea permite que o macho permaneça sobre ela. As caudas aproximam-se ao mesmo tempo em que os membros anteriores do macho são posicionados sobre a carapaça da fêmea. O pênis é então protraído e inserido na cloaca da fêmea. Novamente, o macho pode morder o pescoço da fêmea. A duração total dessas quatro fases pode variar de 15 a 90 minutos, dependendo da receptividade da fêmea. (Molina 1989, 1996). Segundo Astort (1984), durante o acasalamento de Phrynops hilarii pode ainda existir uma fase adicional no comportamento, denominada estabelecimento do par, quando macho e fêmea ficam frente a frente, movendo a cabeça e abrindo a boca. Durante a desova, as fêmeas de Phrynops hilarii em condições naturais podem se posicionar com a carapaça voltada em ângulo que propicie maior incidência de insolação (Bager 1997). O comportamento de acasalamento também foi descrito para outras espécies de Chelidae mantidos em cativeiro, como Platemys platycephala (Harding 1983, Métrailler 2001), Phrynops hilarii (Astort 1984), Mesoclemmys gibbus (Harding e Ewert 1989), Acanthochelys radiolata (Molina 1998, Mocelin 2001) e A. spixii (Molina 1998).

\section{Nidificação e Desova}

O período reprodutivo e demais inferências sobre a reprodução das espécies, como número e tamanho dos ovos, podem ser estabelecidos pela localização de ninhos ou com o auxílio de raios-x (Gibbons e Greene 1979) ou ultra-sons (Schramm et al. 2000) das fêmeas. Radiografias e ultra-sons podem detectar os ovos e, nesse caso, permitir a estimativa do tamanho da ninhada. Ressalte-se que prováveis efeitos deletérios causados aos embriões pela exposição a raios-x são incertos (Hinton et al. 1997).

A nidificação segue uma seqüência de comportamentos, sendo reconhecidas de cinco a sete fases, dependendo da espécie, das quais pelo menos cinco parecem ser comuns a todas as espécies de quelônios (Vanzolini 1967, Foote 1978, Alho e Pádua 1982, Molina 1989, 1998, Bager 1997, Mocelin 2001). Durante a deambulação (ou exploração da área; fase 1), as fêmeas caminham pelo local de nidificação (praia de rio, barrancos ou áreas com cobertura vegetal mais afastada das margens dos rios) como se estivessem à procura de um local propício para a construção do ninho. Nesse comportamento, às vezes podem aproximar a cabeça do solo, como que para examiná-lo olfativamente, ou erguê-la, para melhor visualizar o ambiente ao seu redor. Escolhido o local exato para nidificar, as fêmeas começam então o processo de abertura da cova (fase 2). Durante esse processo, utilizam as patas posteriores para, inicialmente, escavarem uma pequena depressão no solo, que será posteriormente aprofundada e modelada (Pseudemydura umbrina, uma espécie australiana de Chelidae, pode usar os membros anteriores para a escavação do ninho; Kuchling 1993). Durante a abertura da cova, as fêmeas expelem um líquido pela cloaca, umedecendo o substrato, o que pode auxiliar o trabalho de escavação (Guix et al. 1989, Molina 1989, Bager 1997). Os ninhos apresentam uma porção inicial mais estreita, denominada pescoço, seguida de uma porção mais larga e profunda, a câmara de incubação 
(Molina 1989, Bager 1997, Bujes 1998). Terminada a construção da cova, inicia-se a postura dos ovos (fase 3). Conforme a fêmea vai depositando os ovos, esses vão sendo acomodados pelas patas posteriores, de modo a serem posicionados no interior da câmara de incubação. Após o último ovo ter sido depositado, inicia-se a próxima fase do processo de nidificação, o fechamento da cova (fase 4). Os ovos são cobertos e, ao final, geralmente uma fina camada de substrato seco é puxada sobre o local do ninho, para camuflá-lo. Eventualmente, as fêmeas podem compactar a cova esfregando o plastrão sobre o substrato, além de camuflá-lo com material vegetal existente ao redor (Bujes 1998). Na última fase desse comportamento, ocorre o abandono do ninho (fase 5), caracterizado pelo quase sempre imediato retorno da fêmea à água.

A maioria dos dados relativos ao comportamento de nidificação das espécies brasileiras de quelônios não-marinhos refere-se a espécies de Podocnemis (Vanzolini 1967, Foote 1978, Alho e Pádua 1982) ou de cágados mantidos em cativeiro (Guix et al. 1989, Molina 1989, 1996, 1998, Mocelin 2001). Os relatos de nidificação de espécies de Chelidae em condições naturais são raros (Cintra e Yamashita 1989, D'Amato e Morato 1991, Bager 1997, Bujes 1998). De acordo com Molina (1989, 1996, 1998), o comportamento reprodutivo (acasalamento e nidificação) de quelônios parece ser estereotipado e, portanto, observações de cativeiro podem refletir o que acontece na natureza. Entretanto, é possível que variações interespecíficas possam contribuir para que algumas das fases da nidificação mencionadas acima sejam diferenciadas. Por exemplo, espécies de pequeno porte normalmente têm uma ninhada reduzida ( $<5$ ovos) (Figura 2 ), e o que se verifica é que a profundidade dos ninhos para essas espécies é muito pequena (até $5 \mathrm{~cm}$ ) ou que os ovos são depositados na serrapilheira ou entre troncos e raízes (Mittermeier et al. 1978, Pritchard e Trebbau 1984, D'Amato e Morato 1991, Mocelin 2001). Nesses casos, portanto, pelo menos o pescoço não existe, e a câmara de incubação seria a própria cova. Portanto, é possível que, nas espécies pequenas que habitam áreas de florestas (e.g. Hydromedusa maximiliani, Bufocephala vanderhaegei, Acanthochelys spixii, A. radiolata), onde o solo acha-se recoberto por serrapilheira, o ninho se restrinja apenas a uma cova rasa. Aspectos como esse necessitam de maiores investigações.

Geralmente, os ninhos são construídos longe da água, e a seleção do local para a nidificação parece ser bastante criteriosa, sendo que os fatores ambientais que mais influenciam essa escolha são o tipo de substrato, a cobertura vegetal e a inclinação do terreno. Acanthochelys macrocephala nidifica em solos argilosos ou arenosos, em terrenos elevados (entre 16 e 35 $\mathrm{cm})$ e localizados perto $(80 \mathrm{~cm})$ do curso d'água

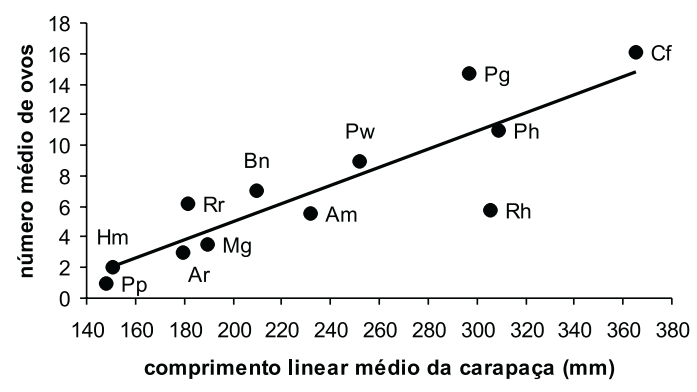

Figura 2 - Relação entre o comprimento médio da carapaça e o tamanho médio da ninhada de espécies brasileiras de Chelidae em condições naturais. Am: Acanthochelys macrocephala (fonte: Cintra e Yamashita 1989), Ar: Acanthochelys radiolata (Mocelin 2001), Bn: Batrachemys nasuta (Medem 1960, Pritchard e Trebbau 1984), Cf: Chelus fimbriatus (Pritchard e Trebbau 1984), Hm: Hydromedusa maximiliani (Yamashita 1990, Guix et al. 1992), Mg: Mesoclemmys gibba (Mittermeier et al. 1978), Ph: Phrynops hilarii (Bager 1997, Bujes 1998), Pp: Platemys platycephala (Pritchard e Trebbau 1984), Pg: Phrynops geoffroanus (Souza 1999, Souza e Abe 2001), Pw: Phrynops williamsi (Rhodin e Mittermeier 1983), Rh: Ranacephala hogei (Moreira 2002), Rr: Rhinemys rufipes (Lamar e Medem 1982). 
(Cintra e Yamashita 1989). Acanthochelys spixii nidifica em áreas abertas com vegetação rasteira, nas proximidades (cerca de $10 \mathrm{~m}$ ) dos rios (D'Amato e Morato 1991). Um ninho de Acanthochelys radiolata foi encontrado sob bromélias em área com pouca incidência de luz (Mocelin 2001). Phrynops geoffroanus nidifica em locais de solo arenoso ou argiloso, coberto por vegetação arbustiva (Medem 1960, Guix et al. 1989, Molina 1989). Phrynops hilarii nidifica preferencialmente em locais de solo arenoso, com predomínio de vegetação herbácea e com boa exposição ao sol, a uma distância média de $80 \mathrm{~m}$ do leito d'água mais próximo (Bager 1997, Bujes 1998). Ninhos de Mesoclemmys gibba podem ser encontrados nas proximidades dos rios (não raro rente às margens), em terrenos argilosos e sombreados (Mittermeier et al. 1978).

Parece haver uma sincronia entre as fases de nidificação e nascimento dos filhotes e as condições climáticas locais. $\mathrm{O}$ que geralmente se observa é que as espécies efetuam a nidificação durante os períodos mais secos do ano, enquanto os nascimentos ocorrem no início ou meados das estações chuvosas. A temperatura do ar também pode exercer alguma influência sobre esse comportamento. Na região do pantanal (MT e MS), Acanthochelys macrocephala parece nidificar entre os meses de abril e maio, um período em que as chuvas estão terminando e o volume de água dos rios começa a diminuir (Rhodin et al. 1984, Cintra e Yamashita 1989). Num padrão bastante semelhante, os filhotes do cágado Hydromedusa maximiliani provavelmente nascem durante o mês de outubro, após a incubação ter transcorrido boa parte do tempo durante a estação seca nas regiões serranas da Mata Atlântica do sudeste do Brasil (Souza 1995, Souza e Abe 1997b). Para o cágado Phrynops geoffroanus, tanto em cativeiro (Guix et al. 1989, Molina 1989, 1998) como em condições naturais no sudeste do Brasil (Souza 1999, Souza e Abe 2001), desovas foram registradas na época mais seca do ano, entre fevereiro e agosto, enquanto os nascimentos parecem ocorrer durante as épocas mais chuvosas, preferencialmente entre dezembro e janeiro. No sul do Brasil, Phrynops hilarii nidifica durante dois períodos distintos, entre fevereiro e maio e entre setembro e dezembro, possivelmente devido ao fato da temperatura do ar ser mais elevada nesses meses (Bager 1997).

A explicação para o comportamento sincronizado entre nidificação e nascimento dos filhotes baseia-se nas condições ambientais favoráveis que os filhotes encontrariam ao eclodirem dos ovos após as chuvas, incluindo ambientes temporários e seguros que os abrigariam, como poças d'água, pequenos lagos ou cursos intermitentes (Guix et al. 1992, Souza e Abe 1997b). Todavia, há que ser ressaltado que tal mecanismo de sincronia deve ser bastante preciso (Alho e Pádua 1982), pois o excesso de umidade no solo durante o processo de incubação dos ovos pode causar sérios danos ao embrião, principalmente quando esse já se encontra em estágio avançado de desenvolvimento (Tucker et al. 1997). Isso significa que eventos naturais, como chuvas repentinas e a conseqüente inundação das margens em épocas de incubação, podem ser danosos e prejudicar a reprodução das espécies em determinadas épocas do ano. De fato, estudos de monitoramento de populações de Podocnemis sextuberculata (Podocnemididae) na região amazônica indicaram que inundações de praias foram responsáveis pela inviabilidade de mais de 50\% dos ninhos (Pezzuti e Vogt 1999). Dessa forma, construções de barragens ou sistemas que modifiquem o regime hidrológico natural dos cursos d'água podem causar sérios danos às espécies, uma vez que a sincronia antes existente é perdida (Tucker et al. 1997).

O número de ovos depositados (tamanho da ninhada) durante a época reprodutiva é variável tanto intra como interespecificamente. Como regra, tem-se uma relação positiva entre o tamanho das fêmeas (comprimento da carapaça ou do plastrão) e o tamanho da ninhada (Molina 1989, Souza 1999, Souza e Abe 2001). Dados compilados para algumas espécies brasileiras de 
Chelidae em condições naturais confirmam essa relação ( $r^{2}=0,77 ; P<0,001$; Figura 2$)$. Variação intra-específica no tamanho da ninhada pode ser observada quando a espécie exibe ampla distribuição geográfica (Iverson et al. 1993, 1997). Todavia, para as espécies brasileiras de cágados, nenhuma atenção foi dada até o momento para esse aspecto.

\section{Alimentação}

A alimentação talvez seja um dos aspectos que mais tem sido abordado e mais consistentemente verificado em estudos de história natural de cágados, embora dados dessa natureza para muitas espécies brasileiras ainda necessitem ser obtidos (Ernst e Barbour 1989, Fachín-Terán et al. 1995, Lima et al. 1997, Souza e Abe 1995, 1997a, 1998, 2000) (Tabela
1). Larvas de insetos que têm parte de seu desenvolvimento na água (e.g. Ephemeroptera, Odonata, Plecoptera, Trichoptera) estão entre os principais recursos alimentares de quelônios aquáticos (Moll 1990, Souza e Abe 1995, 2000, Kennet e Tory 1996, Brandão et al. 2002), além de uma ampla gama de itens de origem animal (incluindo invertebrados, vertebrados e carniça).

De uma maneira geral, as espécies de cágados podem ser consideradas onicarnívoras. Variações intraespecíficas na utilização de recursos alimentares podem ser encontradas, principalmente variações ontogenéticas. Rhinemys rufipes, que habita os igarapés da floresta amazônica, parece ser especialista no consumo de frutos das palmeiras Euterpe precatoria e Socratea exrrhiza, embora itens de origem animal, como tricópteros, crustáceos e lagartos, também contribuam significativamente

Tabela 1 - Conhecimento atual dos aspectos da história natural das espécies brasileiras de Chelidae abordados neste trabalho, considerando-se apenas os dados obtidos em condições naturais. $(+++)$ conhecimento satisfatório, $(++)$ conhecimento razoável, $(+)$ pouco conhecido, $(-)$ desconhecido.

\begin{tabular}{lccc}
\hline Espécies & Padrões de Atividade & Reprodução & Alimentação \\
\hline Acanthochelys macrocephala & + & + & + \\
A. radiolata & - & + & - \\
A. spixii & + & + & + \\
Batrachemys helliostema & - & - & - \\
B. nasuta & - & + & - \\
B. raniceps & - & - & - \\
B. tuberculata & + & - & - \\
Bufocephala vanderhaegei & + & - & ++ \\
Chelus fimbriatus & - & + & ++ \\
Hydromedusa maximiliani & +++ & + & - \\
H. tectifera & + & + & - \\
Mesoclemmys gibba & + & + & + \\
Platemys platycephala & + & + & - \\
Phrynops geoffroanus & ++ & +++ & - \\
P. hilarii & + & + & + \\
. tuberosus & - & - & - \\
williamsi & - & + & + \\
Ranacephala hogei & - & + & + \\
Rhinemys rufipes & + & + & + \\
\hline
\end{tabular}


para a dieta da espécie (Lima et al. 1997). Vegetais também fazem parte da dieta de Batrachemys nasuta (Métrailler 1995). Hábito alimentar especializado em gastrópodos é encontrado entre os cágados Batrachemys raniceps (Fachín-Terán et al. 1995) e Acantochelys macrocephala (Rhodin et al. 1984). Nessas duas espécies, a cabeça dos animais é relativamente grande, uma adaptação para o consumo desse item. Phrynops williamsi apresenta a mandíbula em forma de pá, uma característica que pode representar uma adaptação ao consumo de itens localizados no fundo dos rios (Rhodin e Mittermeier 1983). De acordo com Brandão et al. (2002), invertebrados aquáticos parecem ser os principais componentes da dieta de Acanthochelys spixii. Phrynops geoffroanus é carnívora, consumindo peixes, crustáceos e insetos (Medem 1960, Molina 1989, Dias e Souza no prelo); porém, em locais degradados pela presença humana, aproveita-se de esgoto doméstico (Souza 1999, Souza e Abe 1999, 2000). Fachín-Terán et al. (1995) observaram que insetos e crustáceos são bastante freqüentes na dieta de $P$. geoffroanus durante as épocas mais secas da região amazônica; porém, apenas frutos foram consumidos durante o período das chuvas. Em cativeiro, Hydromedusa tectifera exibe hábito carnívoro, consumindo peixes, anfíbios, lagartos e serpentes (Lema e Ferreira 1990), o mesmo ocorrendo com Mesoclemmys gibba, que também pode aceitar material vegetal (Mittermeier et al. 1978). Hydromedusa maximiliani é carnívora e consome uma ampla variedade de itens, incluindo larvas de insetos, crustáceos, anelídeos, aranhas, anfíbios, lagartos e carniça (Yamashita 1990, Guix et al. 1992, Souza 1995, Souza e Abe 1995, 1997a, 1998). Embora alguns estudos indiquem uma tendência às espécies consumirem determinados itens em detrimento de outros, muito provavelmente esses animais são oportunistas, aproveitando-se da disponibilidade local de alimento. Como exemplo, foi observada variação sazonal na dieta de várias espécies, algumas presas sendo consumidas em certas épocas justamente por serem mais comuns em alguns períodos do ano (Fachin-Teran et al. 1995, Souza 1995, Souza e Abe 1997a).

Podem ocorrer mudanças ontogenéticas na dieta (Souza 1995, Souza e Abe 1998), provavelmente porque uma dieta rica em proteína animal durante a fase jovem possibilita uma maior taxa de crescimento (Hailey e Coulson 1999). Como a fase juvenil dos quelônios está mais sujeita à predação quando comparada à fase adulta (Graham 1984, Gotte 1992), conseguir atingir um maior tamanho o mais rapidamente possível parece ser bastante vantajoso para esses animais. Uma outra conseqüência dessa variação no hábito alimentar é a possibilidade de uma menor sobreposição de nicho, diminuindo-se assim a competição intraespecífica (Fachín-Terán et al. 1995, Souza e Abe 1998). No nível de comunidade, geralmente se observam diferentes padrões de exploração do ambiente por parte dos animais das diversas populações (Moll 1990). Em um estudo envolvendo cinco espécies de quelônios do Rio Guaporé, na região norte do Brasil (RO), Fachín-Terán et al. (1995) constataram que, entre os Chelidae, Phrynops geoffroanus consumiu insetos e crustáceos durante o período chuvoso e sementes e frutos na estação seca, Batrachemys raniceps teve um predomínio de gastrópodos na alimentação e Chelus fimbriatus apresentou um hábito alimentar piscívoro.

Intimamente associadas à alimentação estão as táticas utilizadas pelas diversas espécies de quelônios para a obtenção do alimento (Pritchard 1984, Herrel et al. 2002). Permanecendo imóvel no fundo dos rios e auxiliado por sua coloração críptica, o matamatá (Chelus fimbriatus) espera que a presa entre em seu raio de ação e então, esticando rapidamente o pescoço e abrindo a boca, captura-a (Pritchard 1984, Lemell et al. 2002). Além dessa tática de espreita, C. fimbriatus também pode exibir comportamento ativo de perseguição de presas quando em cativeiro (Holmstrom 1991).

De acordo com Molina (1990) e Molina et al. (1998), após observações detalhadas sobre 
o hábito e o comportamento alimentar de Phrynops geoffroanus e $P$. hilarii mantidos em cativeiro, respectivamente, o comportamento alimentar (desde a detecção até a ingestão total da presa) das espécies brasileiras de cágados pode ser considerado estereotipado, envolvendo forrageio, aproximação, apreensão, dilaceração e ingestão do alimento, etapas nem sempre obrigatórias. Estímulo visual parece ser a principal forma de localização do alimento durante o forrageio. Após a localização, o animal aproxima-se lentamente da presa (se esta estiver imóvel) ou a persegue, caso trate-se de presa viva e ágil. Após o reconhecimento olfativo, utilizado apenas quando se trata de presa imóvel, ocorre a apreensão do alimento por sucção, normalmente por meio de uma rápida projeção da cabeça em direção à presa. Caso a presa tenha um tamanho relativamente grande, ocorre a dilaceração, sendo que o alimento é aparado com uma das mãos. A sucção gradual do alimento ocorre até a ingestão total (Molina, 1990, 1998). Diferentemente de Phrynops spp., as espécies de Hydromedusa, Acanthochelys e Platemys, por serem pouco ágeis, teriam dificuldades em caçar presas de movimento rápido (Molina 1990, Molina et al. 1998). Por terem o pescoço longo, essa limitação de capturar certas presas entre esses Chelidae pode ser contornada pela técnica de caça. Normalmente retraído, o pescoço pode ser rapidamente estendido em direção às presas potenciais, como uma estocada (Pritchard 1984).

\section{Conclusões}

O acúmulo de informações básicas é o primeiro passo para que questões mais abrangentes referentes à história natural dos organismos sejam direcionadas e programas de manejo e conservação possam ser corretamente estabelecidos. A elaboração de cronogramas, métodos e análises deve ser cuidadosamente conduzida para que o máximo de informações possa ser obtido durante o transcorrer dos estudos.
Para a fauna brasileira de cágados, o estudo da história natural das espécies é bastante incipiente, o que significa que muito ainda deve ser feito (Tabela 1). Vários fatores podem estar ligados a essa carência de dados, incluindo desde dificuldades de acesso a algumas áreas de ocorrência das espécies até a falta de estímulo para o desenvolvimento de pesquisas. O resultado da combinação desses fatores é que até mesmo questões mais básicas, como distribuição geográfica, alimentação, atividade e reprodução, são inexistentes para a maioria das espécies. Muitas pesquisas são interrompidas após poucos meses de coleta de dados. Não há continuidade e, dessa maneira, estudos comparativos, ou mesmo de média e longa-duração, são impossibilitados. Não raro, são os exemplos de trabalhos que foram divulgados há mais de 10 anos, freqüentemente 20 ou 30 e, desde então, nada mais foi feito a respeito. Basta uma rápida consulta nas referências bibliográficas do presente trabalho para que esse fato se torne evidente.

Em resumo, a considerável diversidade aliada a uma nítida carência de dados biológicos faz com que os quelônios brasileiros sejam um grupo faunístico muito interessante como fonte de pesquisas. A densidade e a biomassa são altas para muitas espécies, sendo um dos principais grupos a ocuparem os ecossistemas aquáticos (Iverson 1982, Congdon et al. 1986, Souza e Abe 2000). Como salientado anteriormente, a escassez de dados para muitas espécies torna estudos comparativos bastante especulativos. Mesmo para aquelas espécies bastante comuns em algumas localidades (e.g. Phrynops williamsi, Bufocephala vanderhaegei, Acanthochelys macrocephala, Hydromedusa tectifera), estudos conduzidos em condições naturais, quando existem, são bastante incipientes. Alternativamente, o acúmulo de informações obtidas por observação de animais mantidos em cativeiro (Guix et al. 1989, Molina 1989, 1996, 1998) tem possibilitado o preenchimento de várias lacunas referentes à história natural de várias espécies, o que significa que pesquisas ex-situ devem ser incentivadas. 


\section{Agradecimentos}

Agradecimento especial a Flávio B. Molina e Augusto S. Abe, que muito contribuíram para a consolidação de inúmeras idéias tratadas no texto. Flávio B. Molina e um revisor anônimo forneceram comentários valiosos sobre o manuscrito.

\section{Referências Bibliográficas}

Alho, C. J. R. e L. F. M. Pádua. 1982. Reproductive parameters and nesting behavior of the Amazon turtle Podocnemis expansa (Testudinata, Pelomedusidae) in Brazil. Canadian Journal of Zoology 60: 97-103.

Astort, E. D. 1984. Dimorfismo sexual secundario de Phrynops (Phrynops) hilarii (D.y B., 1835) y su conducta reproductora en cautiverio (Testudines Chelidae). Revista del Museo Argentino de Ciencias Naturales "Bernardino Rivadavia" e Instituto Nacional de Investigaciones de las Ciencias Naturales 13: 107-113.

Bager, A. 1997. Aspectos da dinâmica reprodutiva de Phrynops hilarii (Duméril \& Bibron, 1835) (Testudines - Chelidae) no sul do Rio Grande do Sul. Dissertação de Mestrado Não-publicada. Universidade Federal do Rio Grande do Sul, Brasil.

Bjorndal, K. A., A. B. Bolten, T. Dellinger, C. Delgado e H. R. Martins. 2003. Compensatory growth in oceanic loggerhead sea turtles: response to a stochastic environment. Ecology 84: 1237-1249.

Brandão, R. A., G. J. Zerbini, A. Sebben e F. B. Molina. 2002. Notes on distribution and habitats of Acanthochelys spixii and Phrynops vanderhaegei (Testudines, Chelidae) in central Brazil. Boletín de la Asociación Herpetológica Española 13: 11-15.

Brown, G. P, C. A. Bishop e R. J. Brooks. 1994. Growthrate, reproductive output, and temperature selection of snapping turtles in habitats of different productivities. Journal of Herpetology 28: 405-410.

Bujes, C. S. 1998. Atividade de nidificação de Phrynops hilarii Duméril e Bibron (Testudines, Chelidae) na Reserva Biológica do Lami, Rio Grande do Sul. Revista Brasileira de Zoologia 15: 921-928.

Cabrera, M. A. 1998. Las Tortugas Continentales da Sudamérica Austral. BR Cópias, Córdoba, Rep. Argentina. $108 \mathrm{pp}$.

Cintra, R. e C. Yamashita. 1989. Notes on the nesting ecology of Platemys macrocephala in the Brazilian pantanal. Herpetological Review 20: 65-66.
Congdon, J. D., J. L. Greene e J. W. Gibbons. 1986. Biomass of freshwater turtles: a geographic comparision. American Midland.Naturalist 115: 165-173.

D’Amato, A. F. e S. A. A. Morato. 1991. Notas biológicas e localidades de registro de Platemys spixii (Duméril e Bibron, 1835) (Testudines: Chelidae) para o estado do Paraná, Brasil. Acta Biológica Leopoldensia 13: 119-130.

Dias, L. C. e F. L. Souza. Natural History Notes. Phrynops geoffroanus (Geoffroy's Side-necked Turtle). Diet. Herpetological Review. (No prelo.)

Ernst, C. H. e R. W. Barbour. 1989. Turtles of the World. Washington D.C., Smithsonian Institution Press. 313 pp.

Fachín-Terán, A., R. C. Vogt e M. F. S. Gomez. 1995. Food habits of an assemblage of five species of turtles in the Rio Guaporé, Rondônia, Brazil. Journal of Herpetology 29: 536-547.

Foote, R. W. 1978. Nesting of Podocnemis unifilis (Testudines: Pelomedusidae) in the Colombian Amazon. Herpetologica 34: 333-339.

Gibbons, J. W. e J. L. Greene. 1979. X-ray photography: a technique to determine reproductive patterns of freshwater turtles. Herpetologica 35: 86-89.

Gotte, S. W. 1992. Chrysemys picta (Eastern Painted Turtle). Predation. Herpetological Review 23: 80.

Graham, T. E. 1984. Pseudemys rubiventris (Red-bellied Turtle). Predation. Herpetological Review 15: 19-20.

Guix, J. C., M. Salvatti, M. A. Peroni e J. S. Lima-Verde. 1989. Aspectos da reprodução de Phrynops geoffroanus (Schweigger, 1812) em cativeiro (Testudines, Chelidae). Grupo de Estudos Ecológicos, Série Documentos 1: 1-19.

Guix, J. C., J. R. Miranda e V. S. Nunes. 1992. Observaciones sobre la ecologia de Hydromedusa maximiliani. Boletín de la Asociación Herpetológica Española 3: 23-25.

Hailey, A. e I. M. Coulsom. 1999. The growth pattern of the African tortoise Geochelone pardalis and other chelonians. Canadian Journal of Zoology 77: 181193.

Harding, J. H. 1983. Natural History Notes. Platemys platycephala (Twistneck Turtle). Reproduction. Herpetological Review 14: 22.

Harding, J. H. e M. A. Ewert. 1989. Notes on reproductive behavior in captive Phrynops gibbus. Bulletin of the Chicago Herpetological Society 24: 48-49.

Herrel, A., J. C. O'Reilly e A. M. Richmond. 2002. Evolution of bite performance in turtles. Journal of Evolutionary Biology 15: 1083-1094.

Hinton, T. G., P. D. Fledderman, J. E. Lovich, J. D. 
Congdon e J. W. Gibbons. 1997. Radiographic determination of fecundity: is the technique safe for developing turtle embryos? Chelonian Conservation and Biology 2: 409-414.

Holmstrom, W. F. 1991. Further observation on matamata prey herding. Journal of Herpetology 25: 363-364.

Iverson, J. B. 1982. Biomass in turtle populations: a neglected subject. Oecologia 55: 69-76.

Iverson, J. B.1992. A Revised Checklist with Distribution Maps of the Turtles of the World. Privately printed. $363 \mathrm{pp}$.

Iverson, J. B., C. Balgooyan, K. Byrd e K. Lyddan. 1993. Latitudinal variation in clutch size and egg size in turtles. Canadian Journal of Zoology 71: 2448-2461.

Iverson, J. B., C. Griffiths, H. Higgins e A. G. Sirulnik. 1997. Local and geographic variation in the reproductive biology of the snapping turtle (Chelydra serpentina). Herpetologica 53: 96-117.

Kennet, R. e O. Tory. 1996. Diet of two freshwater turtles, Chelodina rugosa and Elseya dentata (Testudines, Chelidae), from the wet-dry tropics of northern Australia. Copeia 1996: 409-419.

Kuchling, G. 1993. Nesting of Pseudemydura umbrina (Testudines: Chelidae): the other way round. Herpetologica 49: 479-487.

Lamar, W. W. e F. Medem. 1982. Notes on the chelid turtle Phrynops rufipes in Colombia (Reptilia: Testudines: Chelidae). Salamandra 18: 305-321.

Lema, T. e M. T. S. Ferreira. 1990. Contribuição ao conhecimento dos Testudines do Rio Grande do Sul (Brasil) - lista sistemática comentada (Reptilia). Acta Biológica Leopoldensia 12: 125-164.

Lemell, P., C. Lemell, P. Snelderwaard, M. Gumpenberger, R. Wochesländer e J. Weisgram. 2002. Feeding patterns of Chelus fimbriatus (Pleurodira, Chelidae). Journal of Experimental Biology 205: 1495-1506.

Lima, A. C., W. E. Magnusson e V. L. Costa. 1997. Diet of the turtle Phrynops rufipes in Central Amazonia. Copeia 1997: 216-219.

Magnusson, W. E., A. C. Lima, V. L. Costa e R. C. Vogt. 1997. Home range of the turtle Phrynops rufipes in an isolated reserve in Central Amazônia, Brazil. Chelonian Conservation and Biology 2: 494-499.

McCord, W. P., M. Joseph-Ouni e W. W. Lamar. 2001. A taxonomic reevaluation of Phrynops (Testudines: Chelidae) with the description of two new genera and a new species of Batrachemys. Revista de Biología Tropical 49: 715-764.

Medem, F. 1960. Informe sobre reptiles colombianos (V). Observaciones sobre la distribucion geografica y ecologia de la tortuga Phrynops geoffroana ssp. en Colombia. Novedads Colombianas 1: 291-300.
Métrailler, S. 1995. Contribution à la connaissance de Phrynops (Batrachemys) n. nasutus en Guyane Française. CITS Bulletin 5: 25-30.

Métrailler, S. 2001. Elevage et reproduction de Platemys platycephala (Schneider, 1792). Manouria 4: 21-32.

Mittermeier, R. A., A. G. J. Rhodin, F. Medem, P. Soini, M. S. Hoogmoed e N. C. Espinoza. 1978. Distribution of the South American chelid turtle Phrynops gibbus, with observations on habitat and reproduction. Herpetologica 34: 94-100.

Mocelin, M. A. O. 2001. Biologia reprodutiva e desenvolvimento dos filhotes de cágado amarelo Acanthochelys radiolata (Mikan, 1820) em cativeiro (Reptilia: Testudines: Chelidae). Dissertação de Mestrado Nãopublicada. Museu Nacional, Universidade Federal do Rio de Janeiro, Brasil.

Molina, F. B. 1989. Observações sobre a biologia e o comportamento de Phrynops geoffroanus (Schweigger, 1812) em cativeiro (Reptilia, Testudines, Chelidae). Dissertação de Mestrado Não-publicada. Universidade de São Paulo, Brasil.

Molina, F. B. 1990. Observações sobre os hábitos e o comportamento alimentar de Phrynops geoffroanus (Schweigger, 1812) em cativeiro (Reptilia, Testudines, Chelidae). Revista Brasileira de Zoologia 7: 319-326.

Molina, F. B. 1996. Mating behavior of captive Geoffroy's side-necked turtles, Phrynops geoffroanus (Testudines: Chelidae). Herpetological Natural History 4: 155160.

Molina, F. B. 1998. Comportamento e biologia reprodutiva dos cágados Phrynops geoffroanus, Acanthochelys radiolata e Acanthochelys spixii (Testudines, Chelidae) em cativeiro. Revista de Etologia (n. esp.): 25-40.

Molina, F. B., M. B. Rocha e L. A. B. M. Lula. 1998. Comportamento alimentar e dieta de Phrynops hilarii (Duméril \& Bibron) em cativeiro (Reptilia, Testudines, Chelidae). Revista Brasileira de Zoologia 15: 73-79.

Moll, D. 1990. Population sizes and foraging ecology in a tropical freshwater stream turtle community. Journal of Herpetology 24: 48-53.

Monteiro, L. P. e C. O. C. Diefenbach. 1987. Thermal regime of Phrynops hilarii (Reptilia, Chelonia). Boletim de Fisiologia Animal, São Paulo 11: 41-50.

Moreira, G. M. 2002. Distribuição, status populacional e conservação do cágado Phrynops hogei (Mertens, 1967) (Tetudines: Chelidae) no Rio Carangola. Dissertação de Mestrado Não-publicada. Universidade Federal de Minas Gerais, Brasil.

Pezzuti, J. C. B. e R. C. Vogt. 1999. Nesting ecology of Podocnemis sextuberculata (Testudines, Pelomedusidae) in the Japurá River, Amazonas, Brazil. Chelonian Conservation and Biology 3: 419-424. 
Pritchard, P. C. H. 1984. Piscivory in turtles, and evolution of the long-necked Chelidae. Symposium of Zoological Society of London 52: 87-110.

Pritchard, P. C. H. e P. Trebbau. 1984. The Turtles of Venezuela. Oxford, Ohio. Society for the Study of Amphibians and Reptiles. 414 pp.

Rhodin, A. G. J. e R. A. Mittermeier. 1983. Description of Phrynops williamsi, a new species of chelid turtle of the South American P. geoffroanus complex. Pp. 58-73 in A. Rhodin e K. Miyata (eds.), Advances in Herpetology and Evolutionary Biology - essays in honor of E.E. Williams. Museum of Comparative Zoology, Cambridge.

Rhodin, A. G. J., R. A. Mittermeier e R. McMorris. 1984. Platemys macrocephala, a new species of chelid turtle from Central Bolivia and the Pantanal region of Brazil. Herpetologica 40: 38-46.

Rowe, J. W., K. A. Coval e K. C. Campbell. 2003. Reproductive characteristics of female midland painted turtles (Chrysemys picta marginata) from a population on Beaver Island, Michigan. Copeia 2003: 326-336.

Schramm, B. G., M. Casares e V. A. Lance. 2000. Ultrasound scanning of ovaries and eggs in Galápagos tortoises, Geochelone nigra, on Santa Cruz Island, Galápagos. Chelonian Conservation and Biology 3: 706-713.

Souza, F. L. 1995. História natural do cágado Hydromedusa maximiliani (Mikan 1820) no Parque Estadual de Carlos Botelho, SP, região de Mata Atlântica (Reptilia, Testudines, Chelidae). Dissertação de Mestrado Nãopublicada. Universidade Estadual Paulista, Rio Claro, Brasil.

Souza, F. L. 1999. Ecologia do cágado Phrynops geoffroanus (Schweigger, 1812) em ambiente urbano poluído (Reptilia, Testudines, Chelidae). Tese de Doutorado Não-publicada. Universidade Estadual Paulista, Rio Claro, Brasil.

Souza, F. L. e A. S. Abe. 1995. Observations on feeding habits of Hydromedusa maximiliani (Testudines:
Chelidae) in southeastern Brazil. Chelonian Conservation and Biology 1: 320-322.

Souza, F. L. e A. S. Abe. 1997a. Seasonal variation in the feeding habits of Hydromedusa maximiliani (Testudines, Chelidae). Boletín de la Asociación Herpetológica Española 8: 17-20.

Souza, F. L. e A. S. Abe. 1997b. Population structure, activity, and conservation of the Neotropical freshwater turtle, Hydromedusa maximiliani, in Brazil. Chelonian Conservation and Biology 2: 521-525.

Souza, F. L. e A. S. Abe. 1998. Resource partitioning by the Neotropical freshwater turtle, Hydromedusa maximiliani. Journal of Herpetolology 32: 106-112.

Souza, F. L. e A. S. Abe. 1999. Fauna urbana: o cágado e a poluição dos rios. Ciência Hoje 25: 59-61.

Souza, F. L. e A. S. Abe. 2000. Feeding ecology, density and biomass of the freshwater turtle, Phrynops geoffroanus, inhabiting a polluted urban river in southeastern Brazil. Journal of Zoology 252: 437-446.

Souza, F. L. e A. S. Abe. 2001. Population structure and reproductive aspects of the freshwater turtle, Phrynops geoffroanus, inhabiting an urban river in southeastern Brazil. Studies on Neotropical Fauna and Environment 36: $57-62$.

Tucker, J. K., F. J. Janzen e G. L. Paukstis. 1997. Response of embryos of the red-eared turtle (Trachemys scripta elegans) to experimental exposure to water-satured substrates. Chelonian Conservation and Biology 2: $338-344$.

Vanzolini, P. E. 1967. Notes on the nesting behavior of Podocnemis expansa in the Amazon valley (Testudines, Pelomedusidae). Papéis Avulsos de Zoologia, São Paulo 20: 191-215.

Vanzolini, P. E., A. M. M. Ramos-Costa e L. J. Vitt. 1980. Répteis das Caatingas. Rio de Janeiro. Academia Brasileira de Ciências. 161 pp.

Yamashita, C. 1990. Natural History Notes. Hydromedusa maximiliani. Ecology. Herpetological Review 21: 19. 